\title{
RESONANCES AND SPECTRAL SHIFT FUNCTION FOR A MAGNETIC SCHRÖDINGER OPERATOR
}

\author{
ABDALLAH KHOCHMAN
}

\begin{abstract}
We consider the 3D Schrödinger operator $H_{0}$ with constant magnetic field and subject to an electric potential $v_{0}$ depending only on the variable along the magnetic field $x_{3}$. The operator $H_{0}$ has infinitely many eigenvalues of infinite multiplicity embedded in its continuous spectrum. We perturb $H_{0}$ by smooth scalar potentials $V=O\left(\left\langle\left(x_{1}, x_{2}\right)\right\rangle^{-\delta_{\perp}}\left\langle x_{3}\right\rangle^{-\delta_{\|}}\right)$, $\delta_{\perp}>2, \delta_{\|}>1$. We assume also that $V$ and $v_{0}$ have an analytic continuation, in the magnetic field direction, in a complex sector outside a compact set. We define the resonances of $H=H_{0}+V$ as the eigenvalues of the non-selfadjoint operator obtained from $H$ by analytic distortions of $\mathbb{R}_{x_{3}}$. We study their distribution near any fixed real eigenvalue of $H_{0}, 2 b q+\lambda$ for $q \in \mathbb{N}$. In a ring centered at $2 b q+\lambda$ with radiuses $(r, 2 r)$, we establish an upper bound, as $r$ tends to 0 , of the number of resonances. This upper bound depends on the decay of $V$ at infinity only in the directions $\left(x_{1}, x_{2}\right)$. Finally, we deduce a representation of the derivative of the spectral shift function (SSF) for the operator pair $\left(H_{0}, H\right)$ in terms of resonances. This representation justifies the Breit-Wigner approximation and implies a local trace formula.
\end{abstract}

Mathematics classification: 35P25, 35J10, 47F05, 81Q10.

Keywords: Electromagnetic Schrödinger operator, Resonances, Embedded eigenvalue, Spectral shift function, Breit-Wigner approximation, Trace formula.

\section{INTRODUCTION}

The resonance theory for non-relativistic particles satisfying the Schrödinger equation has been developed following several approaches. Among them we can mention the analytic dilation (see Aguilar-Combes [1]) or the analytic distortion (see Hunziker [10]) and meromorphic continuation of the resolvent or of the scattering matrix (see Lax-Philips [14] and Vainberg [20]). For Schrödinger operators with constant magnetic field, the resonances can be defined by analytic dilation (only) with respect to the variable along the magnetic field (see Avron-Herbst-Simon [3], Wang [21], Astaburuaga-Briet-Bruneau-Fernández-Raikov [2]) and by meromorphic continuation of the resolvent (see J.F.Bony-Bruneau-Raikov [4]).

The link between the resonances and the spectral shift function (SSF) by the so-called Breit-Wigner approximation has been developed in different situations. Such a representation of the derivative of the spectral shift function related to the resonances, implies trace formulas. In the semi-classical regime we can mention Sjöstrand [18], [19], Petkov-Zworski [15], J.F.Bony-Sjöstrand [5], Bruneau-Petkov [6] and Dimassi-Zerzeri [8] for the Schrödinger operator and 12 for the Dirac operator. In [4], J.-F.Bony, Bruneau and Raikov obtain a Breit-Wigner approximation of the spectral shift function near a Landau level for the 3D Schrödinger operator with constant magnetic field. For the last operator, under more general assumptions, Fernández-Raikov [9] studied the singularities of the spectral shift function at a

Date: October 26, 2018. 
Landau level. These singularities has been analysed at eigenvalues of infinite multiplicity by Astaburuaga, Briet, Bruneau, Fernández and Raikov [2] for a magnetic Schrödinger operator having electric potential depending (only) on the variable along the magnetic field.

In this paper we consider the magnetic Schrödinger operator $H_{0}$ with an electromagnetic field introduced in [2]. We suppose that the magnetic field is constant and that the electric potential $v_{0}$ depends only on the variable $x_{3}$ and is analytic outside a compact set. This operator is remarkable because of the generic presence of infinitely many eigenvalues of infinite multiplicity, embedded in the continuous spectrum of $H_{0}$. We perturb the operator $H_{0}$ by a smooth scalar potential $V$ analytic outside a compact set with respect to the variable $x_{3}$.

The purpose of this work is to define the resonances of the electromagnetic Schrödinger operator $H=H_{0}+V$ for analytic perturbation outside a compact set in the third direction $x_{3}$. We define the resonances for $H$ as the discrete eigenvalues of the non-selfadjoint operator $H_{\theta}$ obtained from the magnetic Schrödinger operator by a general class of complex distortions of $\mathbb{R}_{x_{3}}$. In Section 3, we prove that the discrete eigenvalues of $H_{\theta}$ are the zeros of a regularized determinant $\operatorname{det}_{2}(\cdot)$ which is independent of the distortion. This justifies the definition of the resonances. We calculate the essential spectrum of the distorted operator to determine the sector where we can define the resonances. In Section 4, we establish an upper bound for the number of resonances of $H$ in a domain of size $r \rightarrow 0$ near an embedded eigenvalue of $H_{0}$. The second goal of this work is to obtain a Breit-Wigner approximation for the derivative of the spectral shift function $\xi(\lambda)$ related to the resonances of the operator $H$, as well as a local trace formula (see Section 5 ).

\section{Assumptions And Results}

In this section, we summarize some spectral properties of the 3D Schrödinger operator $H_{0}$ with constant magnetic field $\mathbf{B}=(0,0, b), b>0$ and subject to a non-constant electric field $E=-\left(0,0, v_{0}^{\prime}\left(x_{3}\right)\right)$ depending only on the variable $x_{3}$ (see [2]). We also state the main results. Let

$$
H_{0}=H_{0, \perp} \otimes I_{\|}+I_{\perp} \otimes H_{0, \|},
$$

where $I_{\|}$and $I_{\perp}$ are the identity operators in $L^{2}\left(\mathbb{R}_{x_{3}}\right)$ and $L^{2}\left(\mathbb{R}_{x_{1}, x_{2}}^{2}\right)$ respectively,

$$
H_{0, \perp}:=\left(i \frac{\partial}{\partial x_{1}}-\frac{b x_{2}}{2}\right)^{2}+\left(i \frac{\partial}{\partial x_{2}}+\frac{b x_{1}}{2}\right)^{2}-b, \quad\left(x_{1}, x_{2}\right) \in \mathbb{R}^{2},
$$

is the Landau Hamiltonian shifted by the constant $b$, self-adjoint in $L^{2}\left(\mathbb{R}^{2}\right)$, and

$$
H_{0, \|}:=-\frac{d^{2}}{d x_{3}^{2}}+v_{0}, \quad x_{3} \in \mathbb{R} .
$$

The operator $v_{0}$ is the multiplication operator by an one dimensional scalar potential $v_{0}\left(x_{3}\right)$. We suppose that $v_{0} \in L^{\infty}(\mathbb{R})$ and satisfies

$$
\left|v_{0}\right|=O\left(\left\langle x_{3}\right\rangle^{-\delta_{0}}\right)
$$

with $\langle x\rangle=\left(1+|x|^{2}\right)^{\frac{1}{2}}$ and $\delta_{0}>1$. Then using Weyl theorem, we have

$$
\sigma_{e s s}\left(H_{0, \|}\right)=\sigma_{e s s}\left(-\frac{d^{2}}{d x_{3}^{2}}\right)=[0,+\infty[\text {. }
$$


It is well known that the spectrum of the operator $H_{0, \perp}$ consists of the Landau levels $2 b q, q \in$ $\mathbb{N}:=\{0,1,2 \ldots\}$, and the multiplicity of each eigenvalue $2 q b$ is infinite (see [3]). Consequently, the eigenvalues of $H_{0}$ have the form $2 b q+\lambda$ where $q \in \mathbb{N}$ and $\lambda$ is an eigenvalue of the one dimensional Schrödinger operator $H_{0, \|}=-\frac{d^{2}}{d x_{3}^{2}}+v_{0}\left(x_{3}\right)$. For simplicity, throughout the article we suppose also that

$$
\inf \sigma\left(H_{0, \|}\right)>-2 b .
$$

Note that, (2.6) holds true if $v_{0}>-2 b$. The eigenvalues of $H_{0}, 2 b q+\lambda, q \in \mathbb{N}^{*}$, are embedded in its continuous spectrum $\left[0,+\infty\left[=\cup_{q=0}^{\infty}[2 b q, \infty[\right.\right.$ and are of infinite multiplicity.

Now, we introduce the perturbed operator $H=H_{0}+V$ where $V$ is the multiplication operator by the potential $V(x)$. Assume that $V \in L^{\infty}\left(\mathbb{R}^{3}\right)$ and satisfies

$$
|V(x)|=O\left(\left\langle X_{\perp}\right\rangle^{-\delta_{\perp}}\left\langle x_{3}\right\rangle^{-\delta_{\|}}\right), \quad X_{\perp}=\left(x_{1}, x_{2}\right),
$$

with $\delta_{\perp}>2$ and $\delta_{\|}>1$. We suppose also that $V$ and $v_{0}$ have holomorphic extensions in the magnetic field direction $x_{3}$ in the sector

$$
C_{\epsilon, 0}:=\left\{z \in \mathbb{C} ;|\operatorname{Im}(z)| \leq \epsilon|\operatorname{Re}(z)|, \quad|\operatorname{Re}(z)| \geq R_{0}>0\right\}, \text { for } 0<\epsilon<1,
$$

and satisfy respectively (2.7) and (2.4) for $x_{3} \in C_{\epsilon, 0}$.

For $\theta \in D_{\epsilon} \cap \mathbb{R}$ with $D_{\epsilon}:=\left\{\theta \in \mathbb{C} ;|\theta| \leq r_{\epsilon}:=\frac{\epsilon}{\sqrt{1+\epsilon^{2}}}\right\}$, we denote

$$
H_{\theta}:=\left(I_{\perp} \otimes U_{\theta}\right) H\left(I_{\perp} \otimes U_{\theta}^{-1}\right)=H_{0, \theta}+V_{\theta},
$$

where

$$
H_{0, \theta}:=\left(I_{\perp} \otimes U_{\theta}\right) H_{0}\left(I_{\perp} \otimes U_{\theta}^{-1}\right)=H_{0, \perp} \otimes I_{\|}+I_{\perp} \otimes H_{0, \|}(\theta)
$$

and $H_{0, \|}(\theta)=U_{\theta} H_{0, \|} U_{\theta}^{-1}$ (see (3.2) for the definition of $U_{\theta}$ ). We will prouve in the next section that the operator $H_{\theta}$ has an analytic extension for $\theta \in D_{\epsilon}$.

For $\theta_{0}$ fixed in $D_{\epsilon}^{+}:=D_{\epsilon} \cap\{\theta \in \mathbb{C} ; \operatorname{Im}(\theta) \geq 0\}, q \in \mathbb{N}$ and $r \in \mathbb{R}$, we define

$$
\Gamma_{r, \theta_{0}}:=2 b r+\left(1+\theta_{0}\right)^{-2}[0,+\infty[
$$

and

$$
S_{q, \theta_{0}}:=\bigcup_{q<r<q+1} \Gamma_{r, \theta_{0}} .
$$

The spectrum of $H_{0, \theta_{0}}$ is purely essential and we have

$$
\begin{aligned}
\sigma\left(H_{0, \theta_{0}}\right)=\sigma_{e s s}\left(H_{0, \theta_{0}}\right) & =\bigcup_{q \in \mathbb{N}}\left(2 b q+\sigma\left(H_{0, \|}\left(\theta_{0}\right)\right)\right) \\
& =\bigcup_{q \in \mathbb{N}}\left(\Gamma_{q, \theta_{0}} \cup\left(2 b q+\sigma_{\text {disc }}\left(H_{0, \|}\left(\theta_{0}\right)\right)\right)\right),
\end{aligned}
$$

where $\sigma_{\text {disc }}\left(H_{0, \|}\left(\theta_{0}\right)\right)=\sigma_{\text {disc }}\left(H_{0, \|}\right) \cup\left\{z_{1}, z_{2}, \ldots\right\}, \sigma_{\text {disc }}\left(H_{0, \|}\right)$ denotes the discrete spectrum of $H_{0, \|}$ and $z_{1}, z_{2}, \ldots$ are the complex eigenvalues of $H_{0, \|}\left(\theta_{0}\right)$. In the following, we assume that $\sigma_{\text {disc }}\left(H_{0, \|}\right)=\{\lambda\}$. Note that $\lambda$ is necessarily simple.

The essential spectrum of $H_{\theta}$ coincides with that of $H_{0, \theta}$. We prove also that the discrete spectrum of $H_{\theta}$ in $S_{\theta}=\bigcup_{q \in \mathbb{N}} S_{q, \theta}$ is independent of $\theta$ in $D_{\epsilon}^{+}$(i.e. for two values $\theta_{1}, \theta_{2}$, the discrete spectrum of $H_{\theta_{1}}$ and $H_{\theta_{2}}$ coincide on $S_{\theta_{1}} \cap S_{\theta_{2}}$ ), (see Proposition 3.2). This justifies the following definition. 
Definition 2.1. The resonances of $H$ in $S_{\theta_{0}}$ are the discrete eigenvalues of $H_{\theta_{0}}$. The multiplicity of a resonance $z_{0}$ is defined by

$$
\operatorname{mult}\left(z_{0}\right):=\operatorname{rank} \frac{1}{2 i \pi} \int_{\Gamma_{0}}\left(z-H_{\theta_{0}}\right)^{-1} d z
$$

where $\Gamma_{0}$ is a small positively oriented circle centered at $z_{0}$. We will denote $\operatorname{Res}(H)$ the set of resonances.

Remark 2.1. The resonances of $H$ in $\{z \in \mathbb{C} ; \operatorname{Re}(z)<0\}$ are the real discrete eigenvalues of $H$.

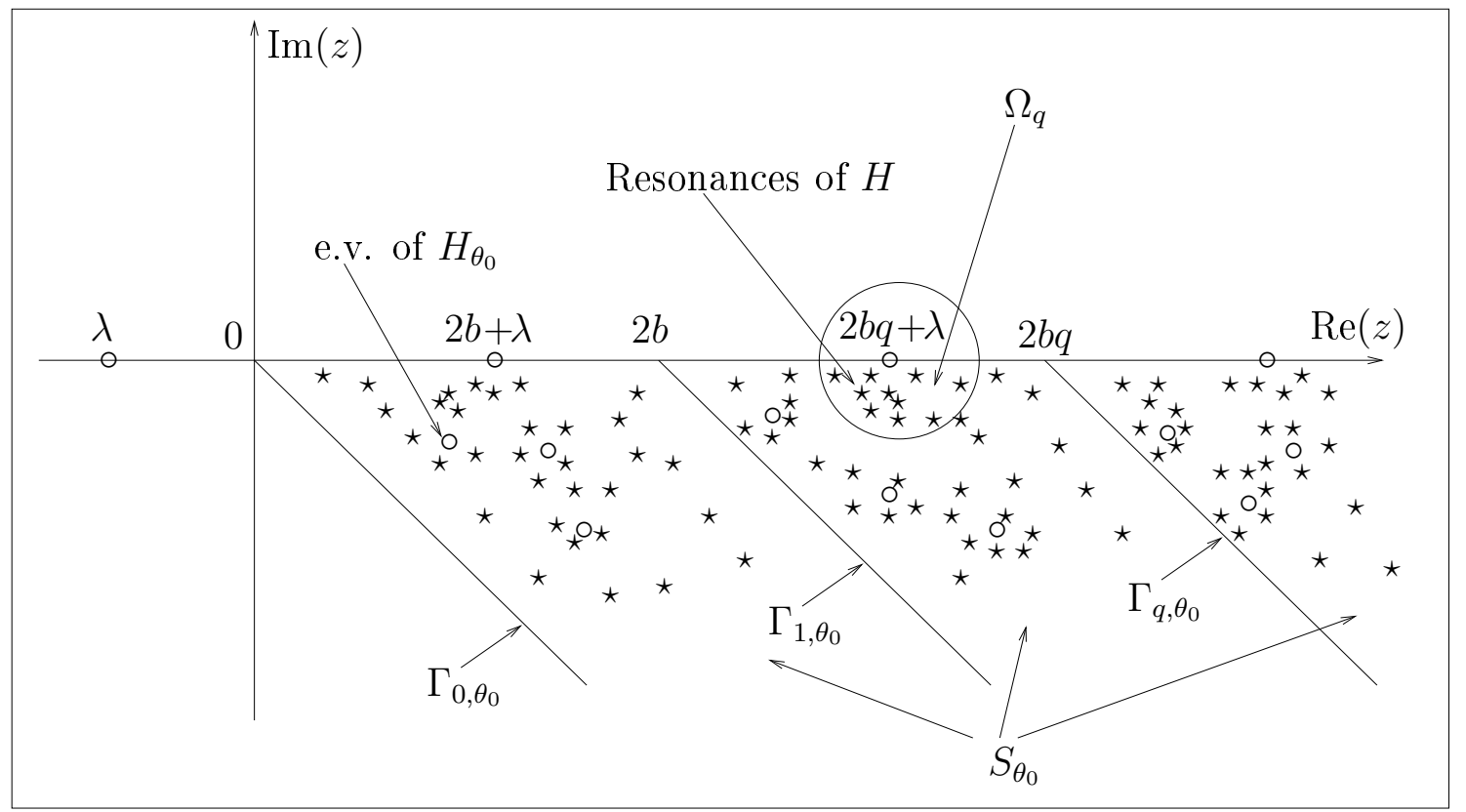

Fig.1. The set $S_{\theta_{0}}$

Now, we state an upper bound as $r \rightarrow 0$ on the number of resonances of $H$ in a ring in $\Omega_{q}$ with radiuses $(r, 2 r)$ and centered at $2 b q+\lambda, q \in \mathbb{N}^{*}$ fixed.

Theorem 2.1. [Upper bound] Suppose that $V$ and $v_{0}$ satisfy the above hypotheses. Then there exist $r_{0}>0$ and $\nu>0$, such that, for any $0<r<r_{0}$,

$$
\#\left\{z \in \operatorname{Res}(H) \cap \Omega_{q} ; r<|z-2 b q-\lambda|<2 r\right\}=O\left(n_{+}\left(r, \nu p_{q} W p_{q}\right)|\ln r|\right),
$$

where $W=\sup _{x_{3} \in C_{\epsilon, 0}}\left|\left\langle x_{3}\right\rangle^{\delta_{\|}} V\right|, p_{q}$ is the orthogonal projection onto $\mathcal{H}_{q}:=\operatorname{ker}\left(H_{0, \perp}-2 b q\right)$ and $n_{+}\left(r, p_{q} W p_{q}\right)$ is the counting function of the eigenvalues larger than $r$ of the Toeplitz operator $p_{q} W p_{q}$. In particular, under our assumption we have always $n_{+}\left(r, p_{q} W p_{q}\right)=O\left(r^{-2 / \delta_{\perp}}\right)$.

The counting function $n_{+}\left(r, p_{q} W p_{q}\right):=\operatorname{rank} \mathbf{1}_{(r,+\infty)}\left(p_{q} W p_{q}\right)$ satisfies asymptotic relations depending on the decay of $W$ at infinity. The following three lemmas give an upper bound of $n_{+}\left(r, p_{q} W p_{q}\right)$ in the case power-like decay, exponential decay, or compact support of $W$, respectively. For more precise results concerning the asymptotic properties, we refer to the cited theorem. 
Lemma 2.1. (Theorem 2.6 of [16]) Let the function $U \in L^{\infty}\left(\mathbb{R}^{2}\right)$ satisfy the estimate

$$
U\left(X_{\perp}\right) \leq C\left\langle X_{\perp}\right\rangle^{-\alpha}, \quad X_{\perp} \in \mathbb{R}^{2},
$$

for some $\alpha>0$. Then for each $q \in \mathbb{N}$, we have

$$
n_{+}\left(r, p_{q} U p_{q}\right)=O\left(r^{-2 / \alpha}\right) .
$$

Lemma 2.2. (Theorem 2.1 of [17]) Let $U \in L^{\infty}\left(\mathbb{R}^{2}\right)$. Assume that

$$
\limsup _{\left|X_{\perp}\right| \rightarrow \infty} \frac{\ln U\left(X_{\perp}\right)}{\left|X_{\perp}\right|^{2 \beta}}<0, \quad X_{\perp} \in \mathbb{R}^{2},
$$

for some $\beta>0$ (with the convention $\ln (u)=-\infty$ if $u \leq 0$ ). Then for each $q \in \mathbb{N}$, we have

$$
n_{+}\left(r, p_{q} U p_{q}\right)=O\left(\varphi_{\beta}(r)\right)
$$

where, for $0<r<e^{-1}$,

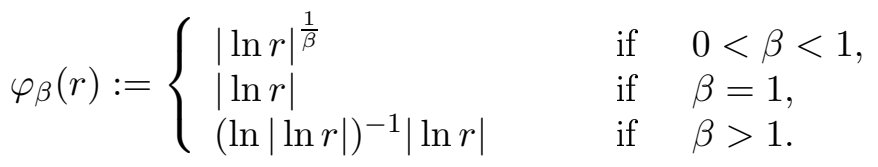

Lemma 2.3. (Theorem 2.4 of [17]) Let $U \in L^{\infty}\left(\mathbb{R}^{2}\right)$. Assume that the support of $U$ is compact. Then for each $q \in \mathbb{N}$, we have

$$
n_{+}\left(r, p_{q} U p_{q}\right)=O\left(\varphi_{\infty}(r)\right)
$$

where, for $0<r<e^{-1}$,

$$
\varphi_{\infty}(r):=(\ln |\ln r|)^{-1}|\ln r|
$$

Now, we study the spectral shift function (SSF) for the pair $\left(H, H_{0}\right)$. The $\operatorname{SSF} \xi(\lambda)$ for a pair of self-adjoint operators $\left(H, H_{0}\right)$ is a distribution in $\mathcal{D}^{\prime}(\mathbb{R})$ whose derivative is

$$
\xi^{\prime}: f \in C_{0}^{\infty}(\mathbb{R}) \longmapsto-\operatorname{tr}\left(f(H)-f\left(H_{0}\right)\right) .
$$

In our case, $|V|^{\frac{1}{2}}\left(H_{0}+i\right)^{-1}$ is in the Hilbert-Schmidt class, (2.16) is well defined and the SSF $\xi(\lambda)$ is a function in $L_{\text {loc }}^{1}(\mathbb{R})$. of

We will see further that the resonances of $H$ in $S_{\theta_{0}}$ are the zeros of the holomorphic extension

$$
z \in\{z \in \mathbb{C}, \operatorname{Im} z>0\} \longmapsto D(z)=\operatorname{det}_{2}\left((H-z)\left(H_{0}-z\right)^{-1}\right)
$$

into $S_{\theta_{0}}$ (see (3.1) for the definition of $\operatorname{det}_{2}$ ). Thus in order to obtain a link between the SSF and the resonances, it will be convenient to introduce the regularized spectral shift function

$$
\xi_{2}(\nu)=\frac{1}{\pi} \lim _{\varepsilon \rightarrow 0^{+}} \arg \operatorname{det}_{2}\left((H-\nu-i \varepsilon)\left(H_{0}-\nu-i \varepsilon\right)^{-1}\right),
$$

whose derivative is the following distribution (see [4])

$$
\xi_{2}^{\prime}: f \in C_{0}^{\infty}(\mathbb{R}) \longmapsto-\operatorname{tr}\left(f(H)-f\left(H_{0}\right)-\left.\frac{d}{d \varepsilon} f\left(H_{0}+\varepsilon V\right)\right|_{\varepsilon=0}\right) .
$$

We will deduce the properties of the SSF from those of the regularized SSF using the relation

$$
\xi^{\prime}=\xi_{2}^{\prime}+\frac{1}{\pi} \operatorname{Im} \operatorname{tr}\left(V_{\theta}\left(H_{0, \theta}-z\right)^{-2}\right) .
$$

We represent now, the derivative of the spectral shift function near $2 b q+\lambda$ as a sum of a harmonic measure related to the resonances and the imaginary part of a holomorphic function. 
Let $\widetilde{\Omega} \subset \subset \Omega$ be open relatively compact subsets of $\mathbb{C} \backslash\{0\}$. We assume that these sets are independent of $r$ and that $\widetilde{\Omega}$ is simply connected. Also assume that the intersections between $\widetilde{\Omega}$ and $\mathbb{R}$ is a non-empty interval $I$.

Theorem 2.2. [Breit-Wigner approximation] We suppose $V$ and $v_{0}$ satisfy the above hypothesis. For $\widetilde{\Omega} \subset \subset \Omega$ and $I$ as above, there exists a function $g$ holomorphic in $\Omega$, such that for $\mu \in 2 b q+\lambda+r I$, we have

$$
\xi^{\prime}(\mu)=\frac{1}{\pi r} \operatorname{Im} g^{\prime}\left(\frac{\mu-2 b q-\lambda}{r}, r\right)-\sum_{\substack{w \in \operatorname{Res}(H) \cap 2 b q+\lambda+r \Omega \\ \operatorname{Im} w \neq 0}} \frac{-\operatorname{Im} w}{\pi|\mu-w|^{2}}-\sum_{w \in \operatorname{Res}(H) \cap 2 b q+\lambda+r I} \delta(\mu-w)
$$

where $g(z, r)$ satisfies the estimate

$$
g(z, r)=O\left(n_{+}\left(r, \nu p_{q} W p_{q}\right)|\ln r|+\widetilde{n}_{1}(r / \nu)+\widetilde{n}_{2}(r / \nu)\right)=O\left(|\ln r| r^{-\frac{2}{\delta_{\perp}}}\right), \quad \nu>0,
$$

uniformly with respect to $0<r<r_{0}$ and $z \in \widetilde{\Omega}$, with $\widetilde{n}_{p}, p=1$, 2 , defined by

$$
\widetilde{n}_{p}(r):=\left\|\frac{p_{q} W p_{q}}{r} \mathbf{1}_{[0, r]}\left(p_{q} W p_{q}\right)\right\|_{p}^{p}, \quad r>0 .
$$

Here, $\|\cdot\|_{p}$ stands for the trace-class norms $(p=1)$ and Hilbert-Schmidt norms $(p=2)$.

Using [4, Corollary1], for $W$ defined above satisfying the assumption of Lemma 2.1 with $\alpha \geq 2$, we have

$$
\widetilde{n}_{p}(r)=O\left(r^{-\frac{2}{\alpha}}\right), p=1,2 .
$$

Finally, if the assumption of Lemma 2.2 or 2.3 hold for $W=U$, we have

$$
\widetilde{n}_{p}(r)=o\left(\varphi_{\beta}(r)\right) \quad r \searrow 0,
$$

the function $\varphi_{\beta}(r)$ being defined in Lemma 2.2 or 2.3 .

As in [15], 6] or [4] and repeating the arguments used in the proof of [4, Corollary 3], we deduce from Theorem 2.1]2.2 the following theorem

Theorem 2.3. [Trace formula] Let $\widetilde{\Omega} \subset \subset \Omega$ be as in Theorem [2.2. Suppose that $f$ is holomorphic on a neighborhood of $\Omega$ and that $\phi \in C_{0}^{\infty}(\Omega \cap \mathbb{R})$ satisfies $\phi=1$ near $\widetilde{\Omega} \cap \mathbb{R}$. Then, under the assumptions of Theorem 2.2, we have the following trace formula

$$
\operatorname{tr}\left((\phi f)\left(\frac{H-2 b q-\lambda}{r}\right)-(\phi f)\left(\frac{H_{0}-2 b q-\lambda}{r}\right)\right)=\sum_{w \in \operatorname{Res}(H) \cap 2 b q+\lambda+r \widetilde{\Omega}} f\left(\frac{w-2 b q-\lambda}{r}\right)+E_{f, \phi}(r)
$$

with

$$
\left|E_{f, \phi}(r)\right| \leq M_{\phi} \sup \{|f(z)|: z \in \Omega \backslash \widetilde{\Omega}, \operatorname{Im} z \leq 0\} \times N_{q}(r),
$$

where $N_{q}(r)=n_{+}\left(r, \nu p_{q} W p_{q}\right)|\ln r|+\widetilde{n}_{1}(r / \nu)+\widetilde{n}_{2}(r / \nu)=O\left(|\ln r| r^{-\frac{2}{\delta_{\perp}}}\right)$, and $M_{\phi}$ depends only on $\phi$. 


\section{Definition of Resonances Via distortion AnALyticity}

In this section, we start with the definition of the deformation for the electromagnetic Schrödinger operator by analytic distortion on $\mathbb{R}_{x_{3}}$. We calculate the essential spectrum of the distorted Schrödinger operator $H_{\theta}$. We prove that the discrete eigenvalues of $H_{\theta}$ are independent of the distortion, this justifies the definition of a resonance as a discrete eigenvalue of the distorted operator $H_{\theta}$. We will also prove that the resonances of $H$ repeated with their multiplicity coincide with the zeros of a regularized determinant $\operatorname{det}_{2}(I+A)$ defined for a Hilbert-Schmidt operator $A$ by

$$
\operatorname{det}_{2}(I+A):=\operatorname{det}\left((I+A) e^{-A}\right),
$$

(see Krein [13]). Let us now introduce the one-parameter family of unitary distortions in the magnetic field direction $x_{3}$ :

$$
U_{\theta} f(x)=J_{\phi_{\theta}(x)}^{\frac{1}{2}} f\left(\phi_{\theta}(x)\right), \quad \theta \in \mathbb{R}, \quad f \in S(\mathbb{R}),
$$

where $\phi_{\theta}(x)=x+\theta g(x), g: \mathbb{R} \longmapsto \mathbb{R}$ is a smooth function and $J_{\phi_{\theta}(x)}=\operatorname{det}\left(I+\theta g^{\prime}(x)\right)$ is the Jacobian of $\phi_{\theta}(x)$. We suppose that $g$ satisfies the assumption

$$
\left(\mathbf{A}_{\mathbf{g}}\right)\left\{\begin{array}{l}
\text { (i) } \sup _{x \in \mathbb{R}}\left|g^{\prime}(x)\right|<1, \\
\text { (ii) } g(x)=0, \text { in the compact set }\left[-R_{0}, R_{0}\right],(\text { see }(2.8)), \\
\text { (iii) } g(x)=x, \text { outside a compact set } K\left(\supset\left[-R_{0}, R_{0}\right]\right) .
\end{array}\right.
$$

We recall that

$$
H_{\theta}:=\left(I_{\perp} \otimes U_{\theta}\right) H\left(I_{\perp} \otimes U_{\theta}^{-1}\right)=H_{0, \theta}+V_{\theta} .
$$

From (2.9) and using Kato's theorem [11, Theorem 4.5.35] we have the following (see also Hunziker [10] for Schrödinger operator and [12, Section 3] for the Dirac operator).

Proposition 3.1. We suppose that the potential $V$ satisfies all the assumptions of Section 2 . Then we have

(i) $\theta \in D_{\epsilon} \longmapsto H_{\theta}=H_{0, \theta}+V_{\theta}$ is an analytic family of type $A$.

(ii) $\sigma_{\text {ess }}\left(H_{\theta}\right)=\sigma_{\text {ess }}\left(H_{0, \theta}\right)=2 b \mathbb{N}+\sigma\left(H_{0, \|}(\theta)\right)$.

Lemma 3.1. The essential spectrum of $H_{0, \|}(\theta)$ is

$$
\sigma_{\text {ess }}\left(H_{0, \|}(\theta)\right)=\left\{\frac{\mu}{(1+\theta)^{2}} \in \mathbb{C} ; \quad \mu \in[0,+\infty[\} .\right.
$$

The rest of the spectrum is

$$
\sigma_{\text {disc }}\left(H_{0, \|}\right) \cup\left\{z_{1}, z_{2}, \ldots\right\}
$$

where $\sigma_{\text {disc }}\left(H_{0, \|}\right)$ denotes the discrete spectrum of $H_{0, \|}$ and $z_{1}, z_{2}, \ldots$ are the complex eigenvalues of $H_{0, \|}(\theta)$.

Remark 3.1. The part $\sigma_{\text {disc }}\left(H_{0, \|}\right) \cup\left\{z_{1}, z_{2}, \ldots\right\}$ of the spectrum of $H_{0, \|}(\theta)$ correspond to eigenvalues with infinite multiplicity of $I_{\perp} \otimes H_{0, \|}(\theta)$.

In the following we fix $q \in \mathbb{N}$ and a compact set $\Omega_{q}$ centered at $2 b q+\lambda$ such that

$$
\Omega_{q} \cap \sigma_{e s s}\left(H_{\theta}\right)=\{2 b q+\lambda\} \text {. }
$$

Repeating arguments in the proof of [4, Proposition 1], 4, Lemma 1] and using the resolvent equation

$$
\left(H_{0}-z\right)^{-1}=\left(H_{0}-v_{0}-z\right)^{-1}\left(I-v_{0}\left(H_{0}-z\right)^{-1}\right),
$$

we obtain the following lemma. 
Lemma 3.2. The operators $V\left(H_{0}-z\right)^{-1}$ and $\partial_{z}\left(V\left(H_{0}-z\right)^{-1}\right)$ are holomorphic on $\{z \in$ $\mathbb{C} ; \operatorname{Im}(z)>0\}$ with values in the Hilbert-Schmidt class $S_{2}$ and in the trace class $S_{1}$ respectively.

Lemma 3.3. The operators $V_{\theta}\left(H_{0, \theta}-z\right)^{-1}$ and $\partial_{z}\left(V_{\theta}\left(H_{0, \theta}-z\right)^{-1}\right)$ are holomorphic for $z \in$ $\Omega_{q} \backslash\{2 b q+\lambda\}$ with values in the Hilbert-Schmidt class $S_{2}$ and in the trace class $S_{1}$ respectively.

Proof. According to (i) of Proposition 3.1 and to the definition of $\Omega_{q}$, the function $z \longmapsto$ $V_{\theta}\left(H_{0, \theta}-z\right)^{-1}$ is analytic for $z \in \Omega_{q} \backslash\{2 b q+\lambda\}$. Moreover, from the resolvent equation, for $z \in \Omega_{q} \backslash\{2 b q+\lambda\}$, we have

$$
V_{\theta}\left(H_{0, \theta}-z\right)^{-1}=V_{\theta}\left(H_{0}-i\right)^{-1}\left(1+\left(H_{0}-H_{0, \theta}+z-i\right)\left(H_{0, \theta}-z\right)^{-1}\right) .
$$

If we denote by $p_{q}$ the orthogonal projection onto $\mathcal{H}_{q}:=\operatorname{ker}\left(H_{0, \perp}-2 b q\right)$ we have

$$
\left(H_{0, \theta}-z\right)^{-1}=\sum_{q \in \mathbb{N}} p_{q} \otimes\left(H_{0, \|}(\theta)+2 b q-z\right)^{-1}, \quad z \in \Omega_{q} \backslash\{2 b q+\lambda\} .
$$

Since

$$
H_{0}-H_{0, \theta}=I_{\perp} \otimes\left(H_{0, \|}-H_{0, \|}(\theta)\right)
$$

the operator $\left(H_{0}-H_{0, \theta}\right)\left(H_{0, \theta}-z\right)^{-1}$ is bounded. Consequently, according to Lemma 3.2 and equation (3.5), we obtain the lemma.

Let us now introduce the function

$$
z \in \Omega_{q} \backslash\{2 b q+\lambda\} \longrightarrow d_{\theta}(z)=\operatorname{det}_{2}\left(I+T_{V, \theta}(z)\right),
$$

with $T_{V, \theta}(z)=V_{\theta}\left(H_{0, \theta}-z\right)^{-1}$. The determinant $d_{\theta}(z)$ is well defined according to Lemma 3.3

Proposition 3.2. Let $V$ and $v_{0}$ as in Section Q. The resonances of $H$ in $\Omega_{q}$ are the zeros of the regularized determinant $d_{\theta}(z)=\operatorname{det}_{2}\left(I+T_{V, \theta}(z)\right)$ in $\Omega_{q} \backslash\{2 b q+\lambda\}$, and are independent on $\theta \in D_{\epsilon}$ such that $\Omega_{q} \cap \sigma_{\text {ess }}\left(H_{\theta}\right)=\{2 b q+\lambda\}$.

If $z_{0}$ is a resonance, there exists a holomorphic function $f(z)$, for $z$ close to $z_{0}$, such that $f\left(z_{0}\right) \neq 0$ and

$$
\operatorname{det}_{2}\left(I+T_{V, \theta}(z)\right)=\left(z-z_{0}\right)^{l\left(z_{0}\right)} f(z),
$$

with $0<l\left(z_{0}\right)=\operatorname{mult}\left(z_{0}\right)$ where $\operatorname{mult}\left(z_{0}\right)$ is the multiplicity of the resonance defined by (2.13).

Proof. Since the operator $H_{0, \theta}$ has no spectrum in $\Omega_{q} \backslash\{2 b q+\lambda\}$, we have

$$
H_{\theta}-z=\left(I+V_{\theta}\left(H_{0, \theta}-z\right)^{-1}\right)\left(H_{0, \theta}-z\right) .
$$

Then, if $z \in \Omega_{q} \backslash\{2 b q+\lambda\}$ is a resonance of $H$ which is by definition a discrete eigenvalue of $H_{\theta}$, the determinant $d_{\theta}(z)=\operatorname{det}_{2}\left(I+V_{\theta}\left(H_{0, \theta}-z\right)^{-1}\right)=\operatorname{det}_{2}\left(I+T_{V, \theta}(z)\right)$ vanishes.

Let us recall that, if $A$ is a bounded operator and $\mathrm{B}$ is a trace class operator on some separable Hilbert space, we have $\operatorname{det}(I+A B)=\operatorname{det}(I+B A)$. Moreover, for $A$ bounded and $B$ Hilbert-Schmidt, we have

$$
\operatorname{det}_{2}(I+A B)=\operatorname{det}_{2}(I+B A) .
$$

Then, the function $d_{\theta}(z)=\operatorname{det}_{2}\left(I+T_{V, \theta}(z)\right)$ coincide with $\operatorname{det}_{2}\left(I+T_{V, 0}(z)\right)=\operatorname{det}_{2}\left(I+V\left(H_{0}-\right.\right.$ $z)^{-1}$ ) for $\theta \in \mathbb{R}, \operatorname{Im} z>0$ and by uniqueness of the extension, it is independent on $\theta \in D_{\epsilon}$. Since the resonances of $H$ are the zero of $d_{\theta}(z)$ the resonances are independents on $\theta \in D_{\epsilon}$. 
In a neighborhood of a zero $z_{0}$ of $d_{\theta}(z)$ with multiplicity $l\left(z_{0}\right)$, we write $d_{\theta}(z)=(z-$ $\left.z_{0}\right)^{l\left(z_{0}\right)} G(z)$, where $G(z)$ is a holomorphic function in a neighborhood of $z_{0}$ with $G\left(z_{0}\right) \neq 0$. Then, by the definition of $l_{0}(z)$,

$$
l_{0}(z)=\frac{1}{2 i \pi} \int_{\Gamma} \partial_{z} \ln \operatorname{det}_{2}\left(1+T_{V, \theta}(z)\right) d z
$$

where $\Gamma$ is a small positively oriented circle centered at $z_{0}$. Further, we have

$$
\partial_{z} \ln \operatorname{det}(1+T(z))=\operatorname{tr}\left((1+T(z))^{-1} \partial_{z} T(z)\right), \quad z \in \Omega_{q}
$$

for any operator-valued holomorphic function $T(z)$ in the trace class $S_{1}$. Therefore,

$$
\partial_{z} \ln \operatorname{det}_{2}\left(1+T_{V, \theta}(z)\right)=\operatorname{tr}\left(\left(1+T_{V, \theta}(z)\right)^{-1} \partial_{z} T_{V, \theta}(z)\right)-\operatorname{tr}\left(\partial_{z} T_{V, \theta}(z)\right) .
$$

According to Lemma 3.3. $\partial_{z} T_{V, \theta}(z)$ is holomorphic in the trace class, then its integral on $\Gamma$ vanishes and (3.8) yields

$$
\begin{aligned}
l_{0}(z) & =\frac{1}{2 i \pi} \int_{\Gamma} \operatorname{tr}\left(\left(H_{\theta}-z\right)^{-1} V_{\theta}\left(H_{0, \theta}-z\right)^{-1}\right) d z \\
& =-\frac{1}{2 i \pi} \int_{\Gamma} \operatorname{tr}\left(\left(H_{\theta}-z\right)^{-1}-\left(H_{0, \theta}-z\right)^{-1}\right) d z \\
& =\operatorname{rank} \frac{1}{2 i \pi} \int_{\Gamma}\left(\left(z-H_{\theta}\right)^{-1}-\left(z-H_{0, \theta}\right)^{-1}\right) d z \\
& =\operatorname{rank} \frac{1}{2 i \pi} \int_{\Gamma}\left(z-H_{\theta}\right)^{-1} d z .
\end{aligned}
$$

In the two latter equalities, we have used that the trace of the projector coincide with its rank and the integral of $\left(H_{0, \theta}-z\right)^{-1}$ on $\Gamma$ vanishes since it is holomorphic in $\Omega_{q}$.

\section{UpPer bound For the Number of RESONANCES NeAR $2 b q+\lambda$}

In this section, we establish an upper bound on the number of resonances in a ring of $\Omega_{q}$ centered at $2 b q+\lambda$ (see (3.4) $)$. For $z \in \Omega_{q}$, we write $z=2 b q+\lambda+\eta$ where $\eta$ is a complex number in a domain centered at 0 and $0<r<|\eta|$. Let $W=\sup _{x_{3} \in C_{\epsilon, 0}}\left|\left\langle x_{3}\right\rangle^{\delta_{\|}} V\right|$. There exists a bounded function $M(x)$ such that

$$
V(x)=W\left(X_{\perp}\right)\left\langle x_{3}\right\rangle^{-2 \delta_{3}} M(x), \text { for } \delta_{3}=\delta_{\|} / 2 .
$$

According to the previous section, the resonances in $\Omega_{q}$ can be identified with the points $z \in \Omega_{q}$ where the determinant $d_{\theta}(z)=\operatorname{det}_{2}\left(I+T_{V, \theta}(z)\right)$ vanishes. Using (3.9) $)$, we have

$$
d_{\theta}(z)=\operatorname{det}_{2}\left(I+\mathcal{T}_{V, \theta}(z)\right)
$$

with

$$
\mathcal{T}_{V, \theta}(z)=W^{\frac{1}{2}} M_{\theta}\left\langle x_{3}\right\rangle_{\theta}^{-\delta_{3}}\left(H_{0, \theta}-z\right)^{-1} W^{\frac{1}{2}}\left\langle x_{3}\right\rangle_{\theta}^{-\delta_{3}}
$$

where $M_{\theta}=M\left(X_{\perp}, \phi_{\theta}\left(x_{3}\right)\right)$ and $\left\langle x_{3}\right\rangle_{\theta}:=U_{\theta}\left\langle x_{3}\right\rangle U_{\theta}^{-1}=\left(1+\left(\phi_{\theta}\left(x_{3}\right)\right)^{2}\right)^{\frac{1}{2}}$.

Using spectral theorem, for $\operatorname{Im} z>0$, we can write

$$
\left(H_{0, \theta}-z\right)^{-1}=\sum_{j \in \mathbb{N}} p_{j} \otimes\left(H_{0, \|}(\theta)-z+2 b j\right)^{-1} .
$$


In order to study the resonances near $2 b q+\lambda$, we split $\mathcal{T}_{V, \theta}(z)$ into two parts:

$$
\mathcal{T}_{V, \theta}(z)=\mathcal{T}_{J, \theta}^{-}+\mathcal{T}_{J, \theta}^{+},
$$

where $\mathcal{T}_{J, \theta}^{-}(z)=\sum_{j \leq J} \mathcal{T}_{j, \theta}$ and $\mathcal{T}_{J, \theta}^{+}=\sum_{j>J} \mathcal{T}_{j, \theta}$ for $J>q$ sufficiently large such that $\left\|\mathcal{T}_{J, \theta}^{+}\right\|<$ $\frac{1}{8}$ and $\left\|\mathcal{T}_{J, 0}^{+}\right\|<\frac{1}{8}$ (for that we use the $h$-pseudo-differential calculus and the spectral theorem). Here,

$$
\mathcal{T}_{j, \theta}=M_{\theta} B_{j} \otimes\left\langle x_{3}\right\rangle_{\theta}^{-\delta_{3}} R_{j, \theta}\left\langle x_{3}\right\rangle_{\theta}^{-\delta_{3}}
$$

with $B_{j}=W^{\frac{1}{2}} p_{j} W^{\frac{1}{2}}$ and $R_{j, \theta}=\left(H_{0, \|}(\theta)-z+2 b j\right)^{-1}$. The operator $\left\langle x_{3}\right\rangle^{-\delta_{3}} R_{j, 0}\left\langle x_{3}\right\rangle^{-\delta_{3}}$ is of class trace (see [2]).

Further, let us decompose the self-adjoint operator $B_{j}$ into a trace-class operator whose norm is bounded by $\varepsilon / 2$ for some $\varepsilon>0$ and an operator of finite-rank independent on $r$, namely

$$
B_{j}=B_{j} \mathbf{1}_{[0, \varepsilon / 2]}\left(B_{j}\right)+B_{j} \mathbf{1}_{] \varepsilon / 2,+\infty[}\left(B_{j}\right) .
$$

Then, for $j \neq q$, we have

$$
\begin{aligned}
\mathcal{T}_{j, \theta} & =M_{\theta} B_{j} \mathbf{1}_{[0, \varepsilon / 2]}\left(B_{j}\right) \otimes\left\langle x_{3}\right\rangle_{\theta}^{-\delta_{3}} R_{j, \theta}\left\langle x_{3}\right\rangle_{\theta}^{-\delta_{3}}+M_{\theta} B_{j} \mathbf{1}_{] \varepsilon / 2,+\infty[}\left(B_{j}\right) \otimes\left\langle x_{3}\right\rangle_{\theta}^{-\delta_{3}} R_{j, \theta}\left\langle x_{3}\right\rangle_{\theta}^{-\delta_{3}} \\
& =\mathcal{T}_{j, \theta}^{<}+\mathcal{T}_{j, \theta}^{>} .
\end{aligned}
$$

Let us now analyze the term $\mathcal{T}_{q, \theta}$. Denote by $p_{\|}(\theta)$ the spectral projection onto $\operatorname{Ker}\left(H_{0, \|}(\theta)-\right.$ $\lambda)$. We have $p_{\|}(\theta) \cdot=\left\langle\cdot, \psi_{\bar{\theta}}\right\rangle \psi_{\theta}$ with $\psi_{\theta}=U_{\theta}^{-1} \psi$ and $\psi$ is an eigenfunction satisfying

$$
H_{0, \|} \psi=\lambda \psi, \quad\|\psi\|_{L^{2}(\mathbb{R})}=1, \quad \psi=\bar{\psi} \text { on } \mathbb{R} .
$$

Then we have, for $\eta=z-2 b q-\lambda$

$$
\begin{aligned}
\mathcal{T}_{q, \theta} & =M_{\theta} B_{q} \otimes\left\langle x_{3}\right\rangle_{\theta}^{-\delta_{3}} R_{q, \theta} p_{\|}(\theta)\left\langle x_{3}\right\rangle_{\theta}^{-\delta_{3}}+M_{\theta} B_{q} \otimes\left\langle x_{3}\right\rangle_{\theta}^{-\delta_{3}} R_{q, \theta}\left(I-p_{\|}(\theta)\right)\left\langle x_{3}\right\rangle_{\theta}^{-\delta_{3}} \\
& =-\frac{1}{\eta} \tau_{q}+\tilde{\mathcal{T}}_{q, \theta},
\end{aligned}
$$

with $\tau_{q}=M_{\theta} B_{q} \otimes\left\langle x_{3}\right\rangle_{\theta}^{-\delta_{3}} p_{\|}(\theta)\left\langle x_{3}\right\rangle_{\theta}^{-\delta_{3}}$. We also have

$$
\begin{aligned}
\tilde{\mathcal{T}}_{q, \theta}= & M_{\theta} B_{q} \mathbf{1}_{[0, \varepsilon / 2]}\left(B_{q}\right) \otimes\left\langle x_{3}\right\rangle_{\theta}^{-\delta_{3}} R_{q, \theta}\left(I-p_{\|}(\theta)\right)\left\langle x_{3}\right\rangle_{\theta}^{-\delta_{3}} \\
& +M_{\theta} B_{q} \mathbf{1}_{] \varepsilon / 2,+\infty[}\left(B_{q}\right) \otimes\left\langle x_{3}\right\rangle_{\theta}^{-\delta_{3}} R_{q, \theta}\left(I-p_{\|}(\theta)\right)\left\langle x_{3}\right\rangle_{\theta}^{-\delta_{3}} \\
= & \tilde{\mathcal{T}}_{q, \theta}^{<}+\tilde{\mathcal{T}}_{q, \theta}^{>} .
\end{aligned}
$$

We denote by $A^{>}(z)=\tilde{\mathcal{T}}_{q, \theta}^{>}+\sum_{j \neq q, j \leq J} \mathcal{T}_{j, \theta}^{>}$, and $A^{<}(z)=\tilde{\mathcal{T}}_{q, \theta}^{<}+\sum_{j \neq q, j \leq J} \mathcal{T}_{j, \theta}^{<}+\mathcal{T}_{J, \theta}^{+}$. Then, we have

$$
\mathcal{T}_{V, \theta}(z)=-\frac{1}{\eta} \tau_{q}+A^{>}(z)+A^{<}(z) .
$$

We decompose the operator $\tau_{q}$ into a trace-class operator whose norm is bounded by $r \nu^{-1}$ for $\nu>0$ and an operator of finite-rank:

$$
\begin{aligned}
\tau_{q} & =M_{\theta} B_{q} \mathbf{1}_{\left[0, r \nu^{-1}\right]}\left(B_{q}\right) \otimes\left\langle x_{3}\right\rangle_{\theta}^{-\delta_{3}} p_{\|}(\theta)\left\langle x_{3}\right\rangle_{\theta}^{-\delta_{3}}+M_{\theta} B_{q} \mathbf{1}_{] r \nu^{-1},+\infty[}\left(B_{q}\right) \otimes\left\langle x_{3}\right\rangle_{\theta}^{-\delta_{3}} p_{\|}(\theta)\left\langle x_{3}\right\rangle_{\theta}^{-\delta_{3}} \\
& =\tau_{q, 1}+\tau_{q, 2} .
\end{aligned}
$$

If we take $\varepsilon$ sufficiently small and $\nu>0$ sufficiently large, we have the two following lemmas 
Lemma 4.1. Let $r_{0}>0$. For $z=2 b q+\lambda+\eta \in \Omega_{q}$ and $0<r<\operatorname{Im} \eta<r_{0}$, we have

$$
\mathcal{T}_{V, \theta}(z)=\mathcal{R}_{\theta}(z)+\mathcal{E}_{\theta}(z)
$$

where the operator $\mathcal{R}_{\theta}(z) \in S_{1}$ is the holomorphic operator defined by

$$
\mathcal{R}_{\theta}(z)=-\frac{1}{\eta} \tau_{q, 2}+A^{>}(z) .
$$

The operator $\mathcal{E}_{\theta}(z) \in S_{2}$ is the holomorphic operator defined by

$$
\mathcal{E}_{\theta}(z)=-\frac{1}{\eta} \tau_{q, 1}+A^{<}(z)
$$

Moreover, $\mathcal{E}_{\theta}(z)$ satisfies the following estimate

$$
\left\|\mathcal{E}_{\theta}(z)\right\|<\frac{3}{4}
$$

Using the limiting absorption principle for $\left\langle x_{3}\right\rangle^{-\delta_{3}} R_{j, 0}\left\langle x_{3}\right\rangle^{-\delta_{3}}, j<q$, the decomposition (4.4) is also available for $\theta=0$, and we have

Lemma 4.2. For $z=2 b q+\lambda+\eta \in \Omega_{q}$ and $0<r<\operatorname{Im} \eta<r_{0}$, we have

$$
\mathcal{T}_{V, 0}(z)=\mathcal{R}_{0}(z)+\mathcal{E}_{0}(z)
$$

with $\mathcal{R}_{0}(z)=\left.\mathcal{R}_{\alpha}(z)\right|_{\alpha=0}$ and $\mathcal{E}_{0}(z)=\left.\mathcal{E}_{\alpha}(z)\right|_{\alpha=0}$. Moreover, $\mathcal{E}_{0}(z)$ satisfies the following estimate

$$
\left\|\mathcal{E}_{0}(z)\right\|<\frac{3}{4}
$$

Proposition 4.1. Let $V$ and $v_{0}$ as in Section . For $0<r<|\eta|<r_{0}$ with $r_{0}$ sufficiently small, $z=2 b q+\lambda+\eta \in \Omega_{q}$ is a resonance of $H$ if and only if $z$ is a zero of

$$
\mathcal{D}_{\theta}(z, r)=\operatorname{det}\left(I+\mathcal{R}_{\theta}(z)\left(I+\mathcal{E}_{\theta}(z)\right)^{-1}\right),
$$

where $\mathcal{R}_{\theta}(z)$ is a class trace operator. Moreover, for $\operatorname{Im} z>0$, the determinant $\mathcal{D}_{\theta}(z, s)$ coincides with

$$
\mathcal{D}_{0}(z, s)=\operatorname{det}\left(I+\mathcal{R}_{0}(z)\left(I+\mathcal{E}_{0}(z)\right)^{-1}\right) .
$$

Proof. By Proposition 3.2, for $r<|\eta|<r_{0}, z$ is a resonance of $H$ if and only if $z$ is a zero of $d_{\theta}(z)=\operatorname{det}_{2}\left(I+\mathcal{R}_{\theta}(z)+\mathcal{E}_{\theta}(z)\right)$. We can write

$$
d_{\theta}(z)=\operatorname{det}\left(I+\mathcal{R}_{\theta}(z)\left(I+\mathcal{E}_{\theta}(z)\right)^{-1}\right) \operatorname{det}\left(\left(I+\mathcal{E}_{\theta}(z)\right) e^{-\mathcal{T}_{V, \theta}(z)}\right) .
$$

According to (4.7), we have $\operatorname{det}\left(\left(I+\mathcal{E}_{\theta}(z)\right) e^{-\mathcal{T}_{V, \theta}(z)}\right) \neq 0$, and then the zeros of $d_{\theta}(z)$ are the zeros of $\mathcal{D}_{\theta}(z, r)$ with the same multiplicity.

Using the theory of $h$-pseudo-differential operators (see [7]), the resolvent $\left(H_{0, \|}(\alpha)-z+\right.$ $2 b j)^{-1}$ is uniformly bounded for $\alpha \in D_{\epsilon}^{+}, j \leq J$ and $\operatorname{Im} z>0$ sufficiently large. Then for $z$ fixed with $\operatorname{Im} z \gg 1, \theta \rightarrow \mathcal{D}_{\theta}(z, r)$ is a holomorphic function on $D_{\epsilon}^{+}$(since the construction of $\mathcal{E}_{\theta}(z)$ is not uniform with respect to $\theta$, this property is not clear for $\operatorname{Im} z>0$ near the real axis). Using that for $\theta \in \mathbb{R}$

$$
\mathcal{D}_{\theta}(z, r)=\operatorname{det}\left(I+U_{\theta} \mathcal{R}_{0}(z)\left(I+\mathcal{E}_{0}(z)\right)^{-1} U_{\theta}^{-1}\right)=\operatorname{det}\left(I+\mathcal{R}_{0}(z)\left(I+\mathcal{E}_{0}(z)\right)^{-1}\right),
$$

the function $\theta \longmapsto \mathcal{D}_{\theta}(z, r)$ is constant on the real axis. Thus, by uniqueness of the extension on $\theta$, the determinant $\mathcal{D}_{\theta}(z, r)$ coincides with $\mathcal{D}_{0}(z, r)$ for $\operatorname{Im} z \gg 1$ and $\theta \in D_{\epsilon}^{+}$. Moreover, 
since for $\theta$ fixed in $D_{\epsilon}^{+}, z \longmapsto \mathcal{D}_{\theta}(z, r)$ and $z \longmapsto \mathcal{D}_{0}(z, r)$ are well defined and holomorphic for $\operatorname{Im} z>0$ (see Lemmas 4.1, 4.2), $\mathcal{D}_{\theta}(z, r)$ coincides with $\mathcal{D}_{0}(z, r)$ for $\operatorname{Im} z>0$.

Since there exists an operator $C: L^{2}\left(\mathbb{R}^{2}\right) \rightarrow L^{2}\left(\mathbb{R}^{2}\right)$ such that $B_{q}=C^{*} C$ and $C C^{*}=$ $p_{q} W\left(X_{\perp}\right) p_{q}$ (see [9] and [4]), then for any $r>0$ we have

$$
n_{+}\left(r, B_{q}\right)=n_{+}\left(r, p_{q} W p_{q}\right),
$$

where, for a compact self-adjoint operator $A$ and $r>0$, we set $n_{+}(r, A)=\operatorname{rank} \mathbf{1}_{(r,+\infty)}(A)$.

Lemma 4.3. For $z=2 b q+\lambda+\eta \in \Omega_{q}$ and $0<r<|\eta|<r_{0}$, there exists $\nu>0$ such that

$$
\mathcal{D}_{\theta}(z, r)=O(1) \exp \left(O\left(n_{+}\left(r, \nu p_{q} W p_{q}\right)+1\right)|\ln r|\right) .
$$

Proof. Since $z \rightarrow A^{>}(z)$ is holomorphic near $z=2 b q+\lambda$ or $\eta=0$ with values in $S_{1}$, for $r_{0}$ sufficiently small, there exist a finite-rank operator $A_{0}^{>}$independent of $z$ and $\tilde{A}^{>}(z)$ holomorphic in $S_{1}$ near $z=2 b q+\lambda$ with $\left\|\tilde{A}^{>}(z)\right\|_{\text {tr }} \leq \frac{1}{8},|\eta| \leq r_{0}$ such that

$$
A^{>}(z)=A_{0}^{>}+\tilde{A}^{>}(z) \text {. }
$$

Since we have $\left\|\tilde{A}^{>}(z)\right\|_{\text {tr }} \leq \frac{1}{8}$, for $0<r<|\eta|<r_{0}$,

$$
\operatorname{det}\left(I+\tilde{A}^{>}(z)\left(I+\mathcal{E}_{\theta}\right)^{-1}\right) \neq 0 \text {. }
$$

It follows that for $0<r<|\eta|<r_{0}$, the zeros of $\mathcal{D}_{\theta}(z, r)$ are the zeros of

$$
D_{\theta}(z, r)=\operatorname{det}\left(I+K_{\theta}(z, r)\right) \text {, }
$$

with

$$
K_{\theta}(z, r)=\left(-\frac{1}{\eta} \tau_{q, 2}+A_{0}^{>}\right)\left(I+\mathcal{E}_{\theta}+\tilde{A}^{>}(z)\right)^{-1} .
$$

We recall that $\tau_{q, 2}=M_{\theta} B_{q} \mathbf{1}_{r \nu^{-1},+\infty[}\left(B_{q}\right) \otimes\left\langle x_{3}\right\rangle_{\theta}^{-\delta_{3}} p_{\|}(\theta)\left\langle x_{3}\right\rangle_{\theta}^{-\delta_{3}}$. Since the rank of the projector $p_{\|}(\theta)$ is equal to 1 , the rank of the operator $K_{\theta}(z, r)$ is bounded by $O\left(n_{+}\left(r \nu^{-1}, B_{q}\right)+1\right)=$ $O\left(n_{+}\left(r, \nu p_{q} W p_{q}\right)+1\right)($ see (4.11) $)$ and its norm is bounded by $O\left(|\eta|^{-1}\right)=O\left(r^{-1}\right)$ (see also Proposition 4.1).

By the properties of $K_{\theta}(z, r)$ for $0<r<|\eta|=|z-2 b q-\lambda|<r_{0}$, we have

$$
D_{\theta}(z, r)=\prod_{j=1}^{O\left(n_{+}\left(r, \nu p_{q} W p_{q}\right)+1\right)}\left(1+\lambda_{j}(z, r)\right)=O(1) \exp \left(O\left(n_{+}\left(r, \nu p_{q} W p_{q}\right)+1\right)|\ln r|\right),
$$

uniformly with respect to $(z, r)$, where $\lambda_{j}(z, r)$ are the eigenvalues of $K_{\theta}(z, r)$ which satisfy $\lambda_{j}(z, r)=O\left(|r|^{-1}\right)$. Since

$$
\mathcal{D}_{\theta}(z, r)=D_{\theta}(z, r) \operatorname{det}\left(I+\tilde{A}^{>}(z)\left(I+\mathcal{E}_{\theta}\right)^{-1}\right),
$$

and the norm of $\operatorname{det}\left(I+\tilde{A}^{>}(z)\left(I+\mathcal{E}_{\theta}\right)^{-1}\right)$ is uniformly bounded, the lemma follows.

Lemma 4.4. For $z=2 b q+\lambda+\eta \in \Omega_{q}$, and $0<r<\operatorname{Im} \eta<r_{0}$, there exists $\nu>0$ such that

$$
\left|\mathcal{D}_{0}(z, r)\right| \geq C \exp \left(-C\left(n_{+}\left(r, \nu p_{q} W p_{q}\right)+1\right)|\ln r|\right),
$$

uniformly with respect to $(z, r)$. 
Proof. Repeating the argument (4.13) in the proof of Lemma 4.3 for $\theta=0$ and using Lemma 4.2, there exist a finite-rank operator $K_{0}(z, r)$ satisfying

$$
\operatorname{rank} K_{0}(z, r)=O\left(n_{+}\left(r, \nu p_{q} W p_{q}\right)+1\right), \quad\left\|K_{0}(z, r)\right\|=O\left(r^{-1}\right),
$$

uniformly with respect to $r<|\eta|<r_{0}$ and an operator $\varepsilon(z)$ such that

$$
\mathcal{D}_{0}(z, r)=\operatorname{det}\left(I+K_{0}(z, r)\right) \operatorname{det}(I+\varepsilon(z))
$$

with $\|\varepsilon(z)\|_{\text {tr }} \leq \frac{3}{4}($ see (4.17) $)$.

Let us now estimate $D_{0}(z, r)^{-1}=\operatorname{det}\left(I+K_{0}(z, r)\right)^{-1}$. For $\operatorname{Im} z>r$, we have

$$
D_{0}(z, r)^{-1}=\operatorname{det}\left(\left(I+K_{0}\right)^{-1}\right)=\operatorname{det}\left(I-K_{0}\left(I+K_{0}\right)^{-1}\right) .
$$

By the construction of $K_{0}$, it satisfies

$$
I+K_{0}=\left(I+\mathcal{T}_{V, 0}(z)\right)\left(I+\widetilde{\mathcal{E}}_{0}\right)^{-1}
$$

with $\widetilde{\mathcal{E}}_{0}$ an operator bounded as $\left\|\widetilde{\mathcal{E}}_{0}\right\|<\frac{7}{8}$ and

$$
\mathcal{T}_{V, 0}(z)=\left.\mathcal{T}_{V, \theta}(z)\right|_{\theta=0}=W^{\frac{1}{2}}\left\langle x_{3}\right\rangle^{-\delta_{3}} M\left(H_{0}-z\right)^{-1}\left\langle x_{3}\right\rangle^{-\delta_{3}} W^{\frac{1}{2}} .
$$

Using the resolvent equation, the operator $I+\mathcal{T}_{V, 0}(z)$ is invertible for $\operatorname{Im} z>r>0$, and

$$
\left(I+\mathcal{T}_{V, 0}(z)\right)^{-1}=I-W^{\frac{1}{2}}\left\langle x_{3}\right\rangle^{-\delta_{3}} M(H-z)^{-1}\left\langle x_{3}\right\rangle^{-\delta_{3}} W^{\frac{1}{2}} .
$$

Then $I+K_{0}$ is invertible for $\operatorname{Im} z>r$ and from the spectral theorem

$$
\left\|\left(I+K_{0}\right)^{-1}\right\|=O\left(1+\left\|W^{\frac{1}{2}}\left\langle x_{3}\right\rangle^{-\delta_{3}} M(H-z)^{-1}\left\langle x_{3}\right\rangle^{-\delta_{3}} W^{\frac{1}{2}}\right\|\right)=O\left(1+\frac{1}{|\operatorname{Im} z|}\right) .
$$

Since the operator $K_{0}$ is of finite-rank $O\left(n_{+}\left(r, \nu p_{q} W p_{q}\right)+1\right)$ and using (4.19) and (4.20), we obtain the lemma.

The following lemma contains a version of the well known Jensen inequality which is suitable for our purposes (see [4] for the proof).

Lemma 4.5. Let $\Omega$ be a simply connected domain of $\mathbb{C}$ and let $g$ be a holomorphic function in $\Omega$ with continuous extension to $\bar{\Omega}$. Assume there exists $z_{0} \in \Omega$ such that $g\left(z_{0}\right) \neq 0$ and $g(z) \neq 0$ for $z \in \partial \Omega$. Let $z_{1}, z_{2}, \ldots, z_{N} \in \Omega$ be the zeros of $g$ repeated according to their multiplicity. For any domain $\Omega^{\prime} \subset \subset \Omega$, there exists $C>0$ such that $N\left(\Omega^{\prime}, g\right)$, the number of zeros $z_{j}$ of $g$ contained in $\Omega^{\prime}$, satisfies

$$
N\left(\Omega^{\prime}, g\right) \leq C\left(\int_{\partial \Omega}|\ln | g(z)|| d z+|\ln | g\left(z_{0}\right)||\right) .
$$

Now, applying this lemma to the function $g(z)=\mathcal{D}_{\theta}(z, r)$, we deduce from (4.12), (4.18) and Proposition 4.1 the upper bound on the number of resonances near $2 b q+\lambda$ stated in Theorem 2.1. 


\section{Spectral shift function and Resonances}

In this section, we represent the derivative of the spectral shift function (SSF) near $2 b q+\lambda$ for $q \in \mathbb{N}$ as a sum of a harmonic measure related to resonances, and the imaginary part of a holomorphic function. As in [15], 6], 8], and [4] such representation justifies the Breit-Wigner approximation and implies a trace formula. We deduce also an asymptotic expansion of the SSF near $2 b q+\lambda$; in the case of $v_{0}=0$, this expansion is given in [4]. For a positive potentials $V$ which decay slowly enough as $\left\|X_{\perp}\right\| \rightarrow \infty$, this expansion yields a remainder estimate for the corresponding asymptotic relations obtained in [9].

In order to obtain such a representation formula, the first step is the factorization of the generalized perturbation determinant. To this end, we need some complex-analysis results due to Sjöstrand, summarized in the following

Proposition 5.1. (see [18, [19]) Let $\Omega$ be an open simply connected domain in $\mathbb{C} \backslash\{0\}$ such that $\Omega \cap \mathbb{R}$ is an interval. Let $z \longrightarrow F(z, h), 0<h<h_{0}$, be a family of holomorphic functions in $\Omega$ containing a number $N(h)$ of zeros. We suppose that,

$$
F(z, h)=O(1) e^{O(1) N(h)}, \quad z \in \Omega,
$$

and for all $\rho>0$ small enough, there exists $C>0$ such that for all $z \in \Omega_{\rho}:=\Omega \cap\{\operatorname{Im} z>\rho\}$ we have

$$
|F(z, h)| \geq e^{-C N(h)} .
$$

Then for each open simply connected subset $\tilde{\Omega} \Subset \Omega$ there exists $g(., h)$ holomorphic in $\tilde{\Omega}$ such that

$$
F(z, h)=\prod_{j=1}^{N(h)}\left(z-z_{j}\right) e^{g(z, h)}, \quad \partial_{z} g(z, h)=O(N(h)), \quad z \in \tilde{\Omega} .
$$

Let $\widetilde{\Omega} \subset \subset \Omega$ be open relatively compact subset of $\mathbb{C} \backslash\{0\}$. We assume that these sets are independent of $r$ and that $\widetilde{\Omega}$ is simply connected. Also assume that the intersections between $\widetilde{\Omega}$ and $\mathbb{R}$ is a non empty interval $I$. With these hypotheses we can obtain the following representation of the regularized spectral shift function near $2 b q+\lambda$.

Theorem 5.1. [Representation formula] Suppose that $V$ and $v_{0}$ satisfy the hypotheses of Section Q For $\widetilde{\Omega} \subset \subset \Omega$ and $I$ as above, there exists a function $g$ holomorphic in $\Omega$, such that for $\mu \in 2 b q+\lambda+r I$, we have

$$
\begin{aligned}
\xi_{2}^{\prime}(\mu)= & \frac{1}{\pi r} \operatorname{Im} g^{\prime}\left(\frac{\mu-2 b q-\lambda}{r}, r\right)-\sum_{\substack{w \in \operatorname{Res}(H) \cap 2 b q+\lambda+r \Omega \\
\operatorname{Im} w \neq 0}} \frac{-\operatorname{Im} w}{\pi|\mu-w|^{2}}-\sum_{w \in \operatorname{Res}(H) \cap 2 b q+\lambda+r I} \delta(\mu-w) \\
& -\frac{1}{\pi} \operatorname{Im} \operatorname{tr}\left(\partial_{z} \mathcal{T}_{V, \theta}(\mu)\right),
\end{aligned}
$$

where $g(z, r)$ satisfies the estimate

$$
g(z, r)=O\left(n_{+}\left(r, \nu p_{q} W p_{q}\right)|\ln r|+\widetilde{n}_{1}(r / \nu)+\widetilde{n}_{2}(r / \nu)\right)=O\left(|\ln r| r^{-\frac{2}{\delta_{\perp}}}\right)
$$

uniformly with respect to $0<r<r_{0}$ and $z \in \widetilde{\Omega}$, with $\widetilde{n}_{p}, p=1,2$, defined by (2.21). 
Proof. First, using the resolvent equation, we have

$$
\operatorname{det}_{2}\left((H-z)\left(H_{0}-z\right)^{-1}\right)=\operatorname{det}_{2}\left(I+T_{V, 0}(z)\right) .
$$

Using (3.9), the last determinant coincides with $d_{\theta}(z)=\operatorname{det}_{2}\left(I+\mathcal{T}_{V, \theta}(z)\right)$ for $\theta \in \mathbb{R}$, where $\mathcal{T}_{V, \theta}(z)$ is defined in (4.1). According to previous section, $\mathcal{T}_{V, \theta}(z)$ is extended on $\theta \in D_{\epsilon}$ and

$$
d_{\theta}(z)=D_{\theta}(z, r) \operatorname{det}\left(\left(I+\tilde{A}^{>}(z)+\mathcal{E}_{\theta}(z)\right) e^{-\mathcal{T}_{V, \theta}(z)}\right)
$$

where $D_{\theta}(z, r)$ is defined by (4.14).

By the properties of $\tilde{A}^{>}(z)$ (see (4.13)), for $\widetilde{K}(z)=\tilde{A}^{>}(z)+\mathcal{E}_{\theta}(z)$, the difference $\mathcal{T}_{V, \theta}(z)-$ $\widetilde{K}(z)=-\frac{1}{\eta} \tau_{q, 2}+A_{0}^{>}$is a finite-rank operator. Using the fact that $\operatorname{det}_{2}(I+B)=\operatorname{det}(I+$ $B) e^{-\operatorname{tr} B}$ for a trace-class operator $B$, we have

$$
\operatorname{det}\left(\left(I+\tilde{A}^{>}(z)+\mathcal{E}_{\theta}(z)\right) e^{-\mathcal{T}_{V, \theta}(z)}\right)=\operatorname{det}_{2}(I+\widetilde{K}(z)) e^{-\operatorname{tr}\left(\mathcal{T}_{V, \theta}(z)-\widetilde{K}(z)\right)},
$$

where $\operatorname{det}_{2}(I+\widetilde{K}(z))$ is a non-vanishing holomorphic function. Since $\tilde{A}^{>}(z)$ is holomorphic in $S_{2}$ and

$$
\left\|\frac{B_{q}}{r} \mathbf{1}_{[0, r]}\left(B_{q}\right)\right\|_{2}^{2}=-\int_{0}^{r} \frac{u^{2}}{r^{2}} d n_{+}\left(u, B_{q}\right)=\widetilde{n}_{2}(r),
$$

we have

$$
\|\widetilde{K}(z)\|_{2}^{2}=O\left(\widetilde{n}_{2}(r / \nu)\right),
$$

which implies that $\left|\operatorname{det}_{2}(I+\widetilde{K}(z))\right|=O\left(\exp \left(\widetilde{n}_{2}(r / \nu)\right)\right)$. Using moreover that $\|\widetilde{K}(z)\|<1$, we have also $|\operatorname{det}(I+\widetilde{K}(z))|^{-1}=O\left(\exp \left(\widetilde{n}_{+}(r / \nu)\right)\right)$. Then there exists $g_{1}(\cdot, r)$ holomorphic on $\Omega$ such that, $\frac{d}{d z} g_{1}(z, r)=O\left(\widetilde{n}_{2}(r / \nu)\right)$, on $\widetilde{\Omega}$, and

$$
\operatorname{det}_{2}(I+\widetilde{K}(z))=e^{g_{1}(z, r)} .
$$

We consider now the functions

$$
F_{\theta}: z \in \Omega \longmapsto D_{\theta}(z, r) .
$$

The functions $F_{\theta}$ are holomorphic in $\Omega$ and $\widetilde{w} \in \Omega$ is a zero of $F_{\theta}$ if and only if $z=2 b d+$ $\lambda+\widetilde{w} r$ is a resonance of $H$. Then applying Proposition 5.1 to $F=F_{\theta}$ with $h=r, N(r)=$ $n_{+}\left(r, \nu p_{q} W p_{q}\right)|\ln r|$, we obtain existence of functions $g_{0}$ holomorphic in $\Omega$ such that for $z \in \Omega$, we have the following factorization:

$$
F_{\theta}=\prod_{w \in \operatorname{Res}(H) \cap 2 b q+\lambda+r \Omega}\left(\frac{z r+2 b q+\lambda-w}{r}\right) e^{g_{0}(z, r)},
$$

with

$$
\frac{d}{d z} g_{0}(z, r)=O\left(n_{+}\left(r, \nu p_{q} W p_{q}\right)|\ln r|\right)
$$

uniformly with respect to $z \in \widetilde{\Omega}$. 
Then by definition of $\xi_{2}$ (see (2.17) $)$, for $\mu \in 2 b q+\lambda+r(\Omega \cap \mathbb{R})$ we obtain

$$
\begin{aligned}
\xi_{2}^{\prime}(\mu)= & \frac{1}{\pi r} \operatorname{Im} \partial_{z}\left(g_{0}+g_{1}\right)\left(\frac{\mu-2 b q-\lambda}{r}, r\right)-\sum_{w \in \operatorname{Res}(H) \cap 2 b q+\lambda+r \Omega} \frac{-\operatorname{Im} w}{\pi|\mu-w|^{2}}-\sum_{w \in \operatorname{Res}(H) \cap 2 b q+\lambda+r I} \delta(\mu-w) \\
+ & \frac{1}{\pi r} \operatorname{Im} \operatorname{tr}\left(\partial_{z} \widetilde{K}\left(\frac{\mu-2 b q-\lambda}{r}\right)\right)-\frac{1}{\pi} \operatorname{Im} \operatorname{Im} \operatorname{tr}\left(\partial_{z} \mathcal{T}_{V, \theta}(\mu)\right) .
\end{aligned}
$$

Then, we conclude the proof of Theorem 5.1 with $g=g_{0}+g_{1}+g_{2}$ taking

$$
g_{2}(z, r)=\operatorname{tr}(\widetilde{K}(z))
$$

which satisfies $\frac{d}{d z} g_{2}(z, r)=O\left(\widetilde{n}_{1}(r / \nu)\right)$.

Lemma 5.1. On $\mathbb{R} \backslash(\{2 b \mathbb{N}+\lambda\} \cup\{2 b \mathbb{N}\})$, for $\theta \in D_{\epsilon}^{+}$, $\operatorname{Im} \theta>0$, we have

$$
\xi^{\prime}=\xi_{2}^{\prime}+\frac{1}{\pi} \operatorname{Im} \operatorname{tr}\left(\partial_{z} T_{V, \theta}(\cdot)\right),
$$

where $T_{V, \theta}(z)=V_{\theta}\left(H_{0, \theta}-z\right)^{-1}$.

Proof. We follow the proof of [4, Lemma 8]. From (2.18), we have only to prove

$$
\operatorname{tr}\left(\left.\frac{d}{d \varepsilon} f\left(H_{0}+\varepsilon V\right)\right|_{\varepsilon=0}\right)=-\frac{1}{\pi} \int_{\mathbb{R}} f(\rho) \operatorname{Im} \operatorname{tr}\left(\partial_{z} T_{V, \theta}(\rho)\right) d \rho
$$

for any $f \in C_{0}^{\infty}(\mathbb{R} \backslash(\{2 b \mathbb{N}+\lambda\} \cup\{2 b \mathbb{N}\}))$. As in [4, Lemma 8], we use the Helffer-Sjöstrand formula and we have

$$
\left.\frac{d}{d \varepsilon} f\left(H_{0}+\varepsilon V\right)\right|_{\varepsilon=0}=\frac{1}{\pi} \int_{\mathbb{C}} \bar{\partial} \widetilde{f}(z)\left(H_{0}-z\right)^{-1} V\left(H_{0}-z\right)^{-1} L(d z),
$$

for $\tilde{f} \in C_{0}^{\infty}\left(\mathbb{R}^{2}\right)$ an almost analytic extension of $f$, (i.e. $\widetilde{f}_{\mid \mathbb{R}}=f$ and $\left.\bar{\partial}_{\lambda} \widetilde{f}(\lambda)=O\left(|\operatorname{Im} \lambda|^{\infty}\right)\right)$ and $L(d z)$ denotes the Lebesgue measure on $\mathbb{C}$.

Let us now define

$$
\sigma_{ \pm}(z)=\operatorname{tr}\left(\left(H_{0}-z\right)^{-1} V\left(H_{0}-z\right)^{-1}\right), \quad \pm \operatorname{Im}(z)>0 .
$$

The functions $\sigma_{ \pm}(z)$ satisfy the relation

$$
\sigma_{-}(z)=\overline{\sigma_{+}(\bar{z})}, \quad \operatorname{Im}(z)<0 .
$$

For $\theta \in \mathbb{R}$, the operator

$$
\left(H_{0}-z\right)^{-1} V\left(H_{0}-z\right)^{-1},
$$

is unitarly equivalent to the operator

$$
\left(H_{0, \theta}-z\right)^{-1} V_{\theta}\left(H_{0, \theta}-z\right)^{-1} \text {. }
$$

Using the cyclicity of the trace, we deduce

$$
\sigma_{ \pm}(z)=\operatorname{tr}\left(\partial_{z} T_{V, \theta}(z)\right), \quad \pm \operatorname{Im}(z)>0, \theta \in \mathbb{R} .
$$

From Lemma 3.3, the function $\theta \longrightarrow \partial_{z} T_{V, \theta}(z)$ is holomorphic on $D_{\epsilon}^{+}$with value in the trace class for $\operatorname{Im}(z)>0$. Then, (5.8) is also available for $\theta \in D_{\epsilon}^{+}$and taking $\operatorname{Im} \theta>0$, $z \longrightarrow \sigma_{+}(z)$ can be extended to $\mathbb{R} \backslash(\{2 b \mathbb{N}+\lambda\} \cup\{2 b \mathbb{N}\})$. According to (5.7), $\sigma_{-}(z)$ satisfies the same property of $\sigma_{+}(z)$. 
Hence, $\left.\frac{d}{d \varepsilon} f\left(H_{0}+\varepsilon V\right)\right|_{\varepsilon=0}$ is of trace class, and

$$
\operatorname{tr}\left(\left.\frac{d}{d \varepsilon} f\left(H_{0}+\varepsilon V\right)\right|_{\varepsilon=0}\right)=\frac{1}{\pi} \int_{\operatorname{Im}(z)>0} \bar{\partial} \widetilde{f}(z) \sigma_{+}(z) L(d z)+\frac{1}{\pi} \int_{\operatorname{Im}(z)<0} \bar{\partial} \widetilde{f}(z) \sigma_{-}(z) L(d z) .
$$

Then the Green formula yields the lemma.

We will deduce Theorem 2.2 from Theorem 5.1 by using the previous lemma and the cyclicity of the trace.

Acknowledgments. The author is grateful to V. Bruneau and J.-F. Bony for many helpful discussions. We also thank the French ANR (Grant no. JC0546063) for the financial support.

\section{REFERENCES}

[1] J. Aguilar and J.-M. Combes, A class of analytic perturbations for one-body Schrödinger Hamiltonians. Comm. Math. Phys. 22 (1971), 269-279.

[2] M.A. Astaburuaga, P. Briet, V. Bruneau, C. Fernandez, G. Raikov, Dynamical resonances and SSF singularities for a magnetic Schrödinger operator. Serdica Math. J. 34 (2008), no. 1, 179-218.

[3] J. Avron, I. Herbst, B. Simon, Schrödinger operator with magnetic field. I. General interactions, Duke Math. J. 45 (1978), 847-883.

[4] J.-F. Bony, V. Bruneau and G. Raikov, Resonances and spectral shift function near the Landau levels. Ann. Inst. Fourier (Grenoble) 57 (2007), no. 2, 629-671.

[5] J.-F. Bony and J. Sjöstrand, Trace formula for resonances in small domains, J. Funct. Anal. 184 (2001), 402-418.

[6] V. Bruneau and V. Petkov, Meromorphic continuation of the spectral shift function, Duke Math. J. 116 (2003), 389-430.

[7] M. Dimassi and J. Sjöstrand, Spectral asymptotics in the Semi-classical Limit, London Math. Soc. Lecture Note Ser.268, Univ.press, Cambridge, 1990.

[8] M. Dimassi and M. Zerzeri, A local trace formula for resonances of perturbed periodic Schrödinger operators, J. Funct. Anal. 198 (2003), 142-159.

[9] C. Fernández, and G. D. Raikov, On the singularities of the magnetic spectral shift function at the Landau levels, Ann. Henri Poincaré 5 (2004), 381-403.

[10] W. Hunziker, Distortion analyticity and molecular resonance curves. Ann. Inst. H. Poincaré Phys. Théor. 45 (1986), no. 4, 339-358.

[11] T. Kato, Perturbation Theory, Springer-Verlag, New York, 1966.

[12] A. Khochman, Resonances and Spectral Shift Function for the semi-classical Dirac operator, Reviews in Mathematical Physics, Vol. 19 No. 10 (2007) 1-45.

[13] M. G. Krein, On perturbation determinants and a trace formula for unitary and self-adjoint operator, (Russian) Dokl. Akad. Nauk SSSR 144 (1962), 268-271.

[14] P.D. Lax and R.S. Phillips, Scattering Theory, Academic Press, New York (1967).

[15] V. Petkov and M. Zworski, Semi-classical estimates on the scattering determinant, Annales H. Poincaré, 2 (2001), 675-711.

[16] G.D. Raikov, Eigenvalue asymptotics for the Schrödinger operator with homogeneous magnetic potential and decreasing electric potential. I. Behaviour near the essential spectrum tips, Commun. P.D.E. 15 (1990), 407-434; Errata: Commun. P.D.E. 18 (1993), 1977-1979.

[17] G.D. Raikov and S. Warzel, Quasi-classical versus non-classical spectral asymptotics for magnetic Schroedinger operators with decreasing electric potentials. Reviews in Mathematical Physics 14 (2002) 1051-1072.

[18] J. Sjöstrand, A trace formula and review of some estimates for resonances, in Microlocal analysis and spectral theory (Lucca, 1996), 377-437, NATO Adv. Sci. Inst. Ser. C Math. Phys. Sci., 490, Dordrecht, Kluwer Acad. Publ.1997.

[19] J. Sjöstrand, Resonances for bottles and trace formulae, Math. Nachr. 221 (2001), 95-149.

[20] B.R. Vainberg, The analytic properties of the resolvent for a certain class of bundles of operators. (Russian) Mat. Sb. (N.S.) 77 (119) 1968 259-296. 
[21] X.P. Wang, Barrier resonances in strong magnetic fields. Partial Differential Equations 17 (1992), no. 9-10, 1539-1566.

E-mail address: Abdallah.Khochman@math.u-bordeaux1.fr

Université Bordeaux I, Institut de Mathématiques, UMR CNRS 5251, 351, Cours de la Libération, 33405 Talence, France 